\title{
Antioxidants in Infection
}

\author{
Gerald T. KEUSCH \\ Division of Geographic Medicine and Infectious Diseases \\ Department of Medicine, New England Medical Center \\ and Tufts University School of Medicine \\ Boston, MA 02111, USA
}

\begin{abstract}
Summary Endogenous oxidation reactions are essential for the normal biochemistry of life and are especially critical for leukocyte microbial killing mechanisms in host defense to infectious diseases. However, reactive oxidative intermediates can damage normal tissues unless kept under antioxidant control. Three selected examples of oxidant-antioxidant systems involved in infectious diseases are discussed, regulation of molecular iron catalyzed oxidations, superoxide scavengers and inhibitors of nitric oxide synthase in septic shock, and the use of glutathione replacement therapy in HIV infection and AIDS. The data suggest that antioxidants, and therapy based on increasing antioxidant potential, have a major impact on clinical infectious diseases.
\end{abstract}

Key Words anti-oxidants infection, oxygen radicals, septic shock, iron, nitric oxide, glutathione.

\section{INTRODUCTION}

The physiological and pathophysiological roles of oxidant stress and the importance of antioxidants in clinical medicine have only just begun to be appreciated in the field of infectious diseases. For example, in a recent Symposium organized by the joint Malnutrition Panels of the U.S. Japan Cooperative Medical Sciences Program (1), a session entitled "Antioxidants in Health and Disease" made mention only of the interactions of vitamin E and malaria, a paradoxical situation in which increased vitamin $\mathrm{E}$ levels protect parasites within infected red cells from oxidant mediated elimination (2). Other references to oxidant-antioxidant systems in infection were lacking. The purpose of this review is to highlight the role of oxidants-antioxidants in infectious diseases by discussing three selected examples of increasing current interest, and by so doing to stimulate further research in this important subject. 


\section{BACKGROUND}

There are two important principles to elaborate before discussing specific examples of oxidant-antioxidant systems in infection. First, antimicrobial host defenses are strongly dependent on induced oxidant stress to the microorganisms causing infection. It has long been known that a burst of leukocyte produced reactive oxygen intermediates (ROI's) and free radicals is essential for antimicrobial host defenses (3). The oxidative burst results in the generation of reactive oxygen species such as $\mathrm{H}_{2} \mathrm{O}_{2}$, the superoxide anion $\left(\mathrm{O}_{2}{ }^{-} \cdot\right)$, singlet oxygen $\left({ }^{1} \mathrm{O}_{2}\right)$, and the hydroxyl radical $(\mathrm{OH} \cdot)$, as well as secondary reaction products such as hypochlorous acid generated from interactions of $\mathrm{H}_{2} \mathrm{O}_{2}$ and $\mathrm{Cl}^{-}$ ion within cells. Patients with genetic diseases in which the capability to activate oxidative mechanisms is impaired, such as chronic granulomatous disease, are known to be at increased risk of infection. In addition, recent data demonstrate that nitric oxide $(\mathrm{NO})$ and $\mathrm{O}_{2}{ }^{-} \cdot$ react to produce peroxynitrite $\left(\mathrm{OONO}^{-}\right)$and the free radical of nitric dioxide $\left(\mathrm{NO}_{2}{ }^{\circ}\right)$ which are essential for macrophage tumoricidal and bactericidal activities in vitro $(4,5)$. While the importance of the latter mechanisms for host defense in vivo remains to be conclusively shown, they appear likely to be of clinical relevance.

Secondly, it is well established that uncontrolled oxidative reactions are harmful to normal tissues, and result in chemical damage to proteins, lipids and nucleic acids (6). Therefore it is absolutely necessary to control the reactions which release ROI's and free radicals by biological antioxidants and scavengers in biological systems. However, this control must be balanced, for, as Olson has noted, "the complete quenching of free radicals in cells also quenches life" (7). It is therefore necessary to think of oxidant stress and antioxidants as a system, in which failure to balance the two may result in disease. In this context, the broader definition of antioxidants proposed by Krinsky ( 8 ) is very appropriate: biological antioxidants are "compounds that protect biological systems against the potentially harmful effects of processes or reactions that can cause excessive oxidations."

\section{IRON AND IRON CONTAINING COMPOUNDS AND IRON BINDING PROTEINS}

Iron, a highly reactive metal, is present in the body in ample quantities. In the normal reduction of molecular oxygen to water, oxygen radicals are formed which, in turn, will react with free iron by the Haber-Weiss reaction to form the highly reactive hydroxyl radical. This is a predictably tissue damaging reaction and uncontrolled, except for the fact that the binding of nearly all free iron into iron centered structures (eg heme) and iron-metalloproteins severely limits the amount of free iron available for these reactions. Iron-metalloproteins comprise various enzymes necessary for life, including those functioning to carry oxygen, to detoxify reactive oxygen species, to replicate DNA, and various proteins designed simply to transport iron in a non-reactive state. Because the 
simultaneous need for iron to serve in its many functional roles (including generation of ROI's for control of infection) and to segregate the metal so that it cannot catalyze excessive tissue damaging oxidations is essential for health, iron metablism is a good example to illustrate the principle of balanced oxidantantioxidant systems.

Much of the clinical literature on iron has focused on the problem of iron deficiency and its treatment with iron (9). There is an expressed concern that iron administration may increase the availability of the metal for use by invading pathogens, especially when circulating iron-binding proteins are reduced and unable to fully bind extracellular iron, such as occurs in patients with proteinenergy malnutrition. In addition, the physiological shift of iron from extracellular to intracellular compartments under the influence of cytokines produced during inflammation and infection has been viewed as a host defense strategy to further restrict access to iron by the infectious agent. The potentially favorable effects of reduced iron availability, however, may be offset by the reports of diminished immune function in iron deficiency states, which would increase host vulnerability to infections (10). While there remains major controversy as to the relative importance of these two different impacts of iron, the balance of evidence that pathogens have efficient mechanisms of obtaining iron, even under the most restrictive of in vivo conditions, and that they use low iron as signal to regulate virulence genes, including those controlling iron access systems, suggests that states of iron deficiency are more harmful to the host than to the pathogen $(10)$.

On the other hand, states of iron excess, such as chronic hemolysis associated with hemoglobinopathies, hemochromatosis, and transfusion siderosis are associated with excessive infection morbidity and mortality. Here too controversy exists about the importance of free iron levels. On the one hand, because the pathogens which emerge under these conditions are often those lacking an effective system of iron acquisition, the availability of extra free iron may be essential for their virulence $(11,12)$. On the other hand, evidence of impaired immune function in iron overload states $(13,14)$ suggests that this condition increases susceptibility to infection, presumably by catalyzing membrane damaging lipid peroxidations which impairs cell function. In effect, a ying-ying situation exists in which either iron deficiency or excess results in impaired immune function and increased infections, with the latter being due to excessive oxidative reactions. Hence, normal iron metabolism is a natural balance of oxidative reactions catalyzed by iron and anti-oxidative iron trapping mechanisms which promotes optimal host defense to infection without damaging the host in the process.

ROI'S AND NITROGEN OXIDE RADICALS IN SEPTIC SHOCK AND SHOCK LUNG

The pathogenesis of septic shock is an example of what may occur when complex regulatory mechanisms are uncontrolled, and represent a setting in 
which "if a little is good, a lot may be worse." A multiplicity of interactive responses are turned on by infection, and attempts to visually present these interactions lead to some of most complicated diagrams ever devised in biology. The reader is referred to a recent review for a much more detailed presentation of these complexities (15). For the purpose of the present discussion, we need only consider a more simplified schema:

INITIATION $\rightarrow$ PROPAGATION $\rightarrow$ INTERACTION $\rightarrow$ DAMAGE

Septic shock is usually initiated by infection with gram negative bacterial pathogens which release large quantities of endotoxin (lipopolysaccharide, LPS), but also occurs during infection by gram positive pathogens which do not contain LPS. One common feature is that LPS and peptidoglycan from the gram positive organisms both result in the release of a similar group of cytokines from leukocytes. Both gram negative and positive pathogens also activate the complement system, a cascade of interacting proteins required to generate key intermediates in the inflammatory response, eg C5a, the activated fragment of the 5th component of complement. These mediators activate leukocyte metabolism and endothelial cell adhesion, and upregulate the production of ROI's and free radicals within phagolysosomes, from increased arachidonate metabolism, and by activation of nitrogen oxide synthase (NOS), increasing the production of inducible NO. There are also important interactions among these mediators, for example the ability of some cytokines to regulate the synthesis of one another, the effects of released platelet activating factor (PAF) and other mediators to increase ROI production by neutrophils and platelets, and the ability of ROI's to react with one another, leading to even more tissue damaging products such as $\mathrm{OH} \cdot, \mathrm{OONO}^{-}$, and hypochlorous acid.

Traditional therapy for the complex pathophysiology of septic shock has depended on antimicrobial therapy to eradicate the infection and pressor agents to maintain tissue perfusion. Unfortunately, the release of biologically active cell wall products from antibiotic damaged or dying organisms, including LPS and peptidoglycan, can make the clinical situation worse initially. Induction of the mediators of inflammation by these microbial constituents can increase the volume of fluids which leak across damaged endothelium, leading to shock lung. In addition, the use of pressors to combat hypotension may actually reduce blood flow to tissues and either directly cause damage or set the stage for a classical ischemia-reperfusion ROI induced damage (16). It is not surprising that traditional therapy has limited effectiveness. Recent attempts to use monoclonal antibodies against LPS to interupt the cycle by inactivating the toxic effects of LPS have improved responses in gram negative septic shock, but only to a limited extent (17). Therefore, further experimental studies have been undertaken to more directly control oxidative damage and reduce the NO mediated vasodilation, which may be critical to the pathogenesis of septic shock (15).

The favorable effects of ROI scavengers and antioxidants in experimental endotoxin shock or $E$. coli sepsis has provided the most compelling evidence for the key role of oxy-radicals in the pathogenesis of septic shock. For example, 
Yoshikawa has shown that either superoxide dismutase (SOD) or catalase reduce the rapid hypotensive effects of LPS injections (18), and Broner et al. have reported that SOD, but not $\mathrm{N}$-acetyl cysteine (NAC), reduces endotoxin induced mortality. However, both confirmatory $(19,20)$ and contradictory $(21,22)$ data have been published. Some of the discrepancies may be due to the use of different models (23) and the different pharmacology and pharmacokinetics of the inhibitory agents employed, and there remains much more work to be done. In particular, little direct evidence has been obtained thus far in humans and further study is both required and warranted.

A parallel set of studies has begun to evaluate the role of nitric oxide (NO) in shock. This is a stable, freely diffusible, free radical gas derived from the oxidation of arginine by the enzyme nitric oxide synthase (or NOS), which appears to be a major regulator of normal vascular tone (24). NO is now known to be identical to endothelial-derived relaxing factor, and it is the active metabolite of nitroglycerin and other organic nitrate coronary artery dilators. It binds to the iron heme center of guanyl cyclase and activates production of cGMP which, in turn, regulates a c-GMP dependent protein kinase leading to vascular smooth muscle relaxation. Neutrophils and macrophages contain a distinct, inducible NOS, which is activated by cytokines to produce NO. Because cytokines also activate oxidative reactions leading to superoxide production, and $\mathrm{NO}$ is attacked by $\mathrm{O}_{2}{ }^{-}$. to form peroxynitrite, which decomposes to highly reactive radicals such as $\mathrm{OH}^{-}$and $\mathrm{NO}_{2}{ }^{-}$, a highly microbicidal milieu develops. Overproduction of NO, especially at an endothelial site, could theoretically lead to inappropriate vasorelaxation and a drop in blood pressure.

Can this happen in septic shock? This possibility has been suggested (25), in part because C5a activation of neutrophils leads to the expression of surface receptors mediating neutrophil attachment to vascular endothelium. Several experimental studies have shown that NOS inhibitors greatly affect shock caused by LPS infusion. Thus, use of $N^{\mathrm{G}}$-methyl-L-arginine (L-NMA) in a dog model of endotoxemia (which resulted in a 33\% drop in peripheral vascular resistance and a $54 \%$ decrease in systolic blood pressure) led to restoration of normal values within $1.5 \mathrm{~min}$ of adminstration of the drug (26). In a rodent model, a dose dependent effect of L-NMA was reported, in which $3 \mathrm{mg} / \mathrm{kg}$ L-NMA was ineffective, $30 \mathrm{mg} / \mathrm{kg}$ reversed endotoxin induced shock, and $300 \mathrm{mg} / \mathrm{kg}$ accelerated the fall in blood pressure initiated by endotoxin infusion (27).

Similar findings have been reported when the shock syndrome was induced by a cytokine, tumor necrosis factor (TNF) (28). This may be the pathway by which glucocorticoids exert an effect in septic shock, since the transcriptional activation of both TNF and inducible NOS is prevented by steroids (29). Two patients with refractory septic shock have recently been reported who received treatment with NOS inhibitors, either L-NMA or $N^{\mathrm{G}}$-nitro-L-arginine methyl ester (L-NAME) (30). In both patients a rapid dose-dependent rise in blood pressure was observed in association with a rise in systemic vascular resistance. 
Continuous infusion of L-NAME in one patient allowed blood pressure maintenance with reducing doses of noradrenaline, and by $48 \mathrm{~h}$ both drugs could be stopped. However, this patient died 2 days later of "recurrent intraabdominal sepsis, adult respiratory distress syndrome, and disseminated intravascular coagulation" (DIC). This has raised the possibility that NOS inhibitors are two edged swords, including the improvement of blood pressure in septic shock, but also perhaps resulting in harmful vasoconstriction in the visceral circulation leading to bacterial translocation across a damaged bowel, coronary artery constriction leading to compromised cardiac function and lowered cardiac output, platelet aggregation and promotion of DIC, and reduction in $\mathrm{NO}$ dependent microbicial activity (31).

That there is a complex and perhaps dual role of NO in septic shock is also indicated by experiments in which infusion of NOS inhibitors or administration of NO or arginine have improved outcomes in various shock models (32-34). There is also the theorectical possibility, supported by limited clinical information, that inhalation of NO can result in vasodilation of pulmonary vessels in ventilated segments of the lung during adult respiratory distress syndrome (ARDS) associated with sepsis (35). In this manner, oxygen exchange across ventilated regions of the lung might be preferentially improved, with significant clinical improvement. Since $\mathrm{NO}$ does not react readily with $\mathrm{O}_{2}$ to form toxic products, there seems little chance of local damage in this setting. However, the findings suggest that inhibition of NOS could worsen established ARDS in septic patients. These data make it clear that human studies need to be cautiously and carefully done, and that a balance in NO and ROI metabolism may be essential to optomize outcomes in septic shock.

On the other hand, some data suggest that ARDS may be the consequence of local production of ROI's and free radicals. A necessary and critical feature of ARDS, a syndrome characterized by increased permeability of small pulmonary vessels leading to pulmonary edema, refractory hypoxemia, and respiratory failure, appears to be the recruitment of neutrophils to the lung and the production of ROI's during activation of the cells (36). In one study, administration of superoxide dismutase conjugated to polyethylene glycol (which lengthens the half life of the enzyme and appears to increase its uptake by vascular endothelium) to guinea pigs with lung injury secondary to experimental E. coli sepsis significantly attenuated the damage (37). Another study has reported depressed levels of alveolar fluid glutathione levels in patients with ARDS (38), which suggests that antioxidant deficiency may contribute to lung damage in sepsis. This is consistent with prior experimental studies showing that administration of oxidant generating systems into the rabbit lungs in vitro or in vivo will result in an experimental form of ARDS resembling that seen in human patients $(39,40)$, and with recent reports that expiratory gas condensates of patients with ARDS contains measurable levels of $\mathrm{H}_{2} \mathrm{O}_{2}(41,42)$. The potential interaction of reactive oxygen species and $\mathrm{NO}$ to damage the lung, and the ability of systemic or inhaled antioxidants (such as $N$-acetyl cysteine) to 
prevent the oxidant mediated damage in ARDS, especially if NO inhalation therapy is likely to be employed, should be thoroughly studied.

\section{GLUTATHIONE DEFICIENCY IN THE ACQUIRED IMMUNODEFICIENCY SYNDROME}

Selenium nutrition was first investigated in patients with AIDS idiopathic cardiomyopathy in 1986 because of the reported relationship of selenium deficiency and cardiomyopathy described in Keshan Disease in China (43). Clinical improvement with the oral administration of selenium salts was described the following year (44). Two years later, reduced systemic thiol or glutathione (GSH) levels were reported in AIDS patients $(45,46)$, and subsequently, reduced intracellular GSH levels were detected in T lymphocytes, both CD4+ and CD8+ (47). While the extent of the median GSH reduction was modest, in the range of $60 \%$ of normal, this was actually due to the loss of a subset of CD4 cells with a high GSH level and not just a reduction in the GSH content of all $\mathrm{T}$ cells (48).

The potential significance of these findings has not been ignored, and increasing evidence for an inverse relationship between intracellular CD4 antioxidant capacity and replication of HIV has been reported. In vitro studies have shown that certain cytokines stimulate HIV replication (49), and that GSH, GSH-esters, or $N$-acetyl cysteine inhibit this event (50). Cytokine-stimulated upregulation of HIV procedes via the activation of a nuclear transcription factor, NFKB (51), responding to ROI's produced during cytokine stimulation (52). It was therefore rational to attempt to inhibit this process by exogenous antioxidant thiols, such as $N$-acetylcysteine, which in fact prevents the activation of NFKB by either phorbol esters or TNF (53). While the function of high GSH CD4 + cells is not clear, or even if they represent a distinctive subset of CD4 + cells, GSH deficiency has been shown to reversibly impair several immune responses in which these cells participate, including $\mathrm{T}$ cell proliferation and differentiation, MLR, CTL and NK activity (50). Herzenberg and colleagues have shown that GSH and CD4 cell number vary independently, however in as much as lowered GSH levels are observed even in asymptomatic HIV + individuals (48), an influence of low levels may be exerted early in the course of the disease. Since the progression of asymptomatic HIV infection to AIDS is associated with a burst of virus proliferation and is accompanied by recurrent infections, with both ordinary as well as opportunistic pathogens, it is certainly reasonable to propose that oxidant stimuli generated by cytokine activation during these infections can directly contribute to the upregulation of the virus, especially if unopposed by adequate antioxidant capacity. If this is so, then restoration of antioxidant capacity might have a salutory effect, reduce virus replication, and delay clinical advance of HIV infection towards AIDS (48). Studies are currently under way to evaluate this possibility. If the hypothesis is proven correct, it will propel antioxidant therapy to the fore in HIV, and, no doubt, will encourage similar investigations in other infectious diseases. 


\section{REFERENCES}

1) Olson, J. A., ed. (1992): Proceedings of a conference on molecular and comparative nutrition, bethesda, MD, July 22-24, 1991. Proc. Soc. Exp. Bio. Med., 200, $147-276$.

2) Levander, O. A. (1992): Selenium and sulfur in antioxidant protective systems: Relationships with vitamin E and malaria. Proc. Soc. Exp. Biol. Med., 200, 255-259.

3) Cohen, M. S., Brittigan, B. E., Hassett, D. J., and Rosen, G. M. (1988): Phagocytes, $\mathrm{O}_{2}$ reduction, and hydroxyl radical. Rev. Infect. Dis., 10, 1088-1096.

4) Hibbs, J. B., Jr., Taintor, R. R., and Vavrin, V. (1987): Macrophage cytotoxicity: role for L-arginine deiminase and imino nitrogen oxidation to nitrite. Science, $\mathbf{2 3 5}$, $473-476$.

5) Nathan, C. F., and Hibbs, J. B., Jr. (1991): Role of nitric oxide synthesis in macrophage antimicrobial activity. Curr. Opin. Immunol., 3, 65-70.

6) Diplock, A. T. (1991): Antioxidant nutrients and disease prevention: an overview. Am. J. Clin. Nutr., 53, 189S-193S.

7) Olson, J. A. (1992): Antioxidants in health and disease: overview. Proc. Soc. Exp. Biol. Med., 200, 245-247.

8) Krinsky, N. (1992): Mechanism of action of biological antioxidants. Proc. Soc. Exp. Biol. Med., 200, 248-254.

9) Weinberg, E. S. (1984): Iron withholding: a defense against infection and neoplasia. Physiol. Rev., 64, 65-102.

10) Keusch, G. T. (1990): Micronutrients and susceptibility to infection. Ann. N. Y. Acad. Sci., 587, 181-188.

11) Green, N. S. (1992): Yersinia infections in patients with homozygous betathalassemia assoicated with iron overload and its treatment. Ped. Hematol. Oncol., 9, 247-254.

12) Bullen, J. J., Spalding, P. B., Ward, C. G., and Gutteridge, J. M. (1991): Hemochromatosis, iron and septicemia caused by Vibrio vulnificus. Arch. Int. Med., 151, 1606-1609.

13) De Sousa M. (1989): Immune cell functions in iron overload. Clin. Exp. Immunol., 75, $1-6$.

14) Cantinieaux, B., Hariga, C., Ferster, A., De Maertelaere, E., Toppet, M., and Fondu, P. (1987): Neutrophil dysfuntions in thalassemia major: the role of cell iron overload. Eur. J. Haematol., 39, 28-34.

15) Glauser, M. P., Zanetti, G., Baumgartner, J. D., and Cohen, J. (1991): Septic shock: pathogenesis. Lancet, 338, 732-736.

16) McCord, J. M. (1985): Oxygen-derived free radicals in post ischemic tissue injury. New Eng. J. Med., 312, 159-163.

17) Ziegler, E. J., Fisher, C. J., Jr., Sprung, C. L., Straube, R. C., Sadoff, J. C., Foulke, G. E., Wortel, C. H., Fink, M. P., Dellinger, R. P., and Teng, N. N. (1991): Treatment of gram-negative septic shock with HA-1A human monoclonal 
antibody against endotoxin - a randomized, double-blind, placebo-controlled trial. New Eng. J. Med., 324, 429-436.

18) Yoshikawa, T. (1990): Oxy radicals in endotoxin shock. Methods Enzymol., 186, 660-665.

19) Kunimoto, F., Morita, T., Ogawa, R., and Fujita, T. (1987): Inhibition of lipid peroxidation improves survival rates of endotoxemic rats. Circ. Shock, 21, 15-22.

20) Warner, B. W., Hassegren, P. O., and Fischer, J. E. (1986): Effect of allopurinol and superoxide dismutase on survival rate in rats with sepsis. Curr. Surg., 43, 292-293.

21) McKechnie, K., Furman, B. L., and Parratt, J. R. (1986): Modification by oxygen free radical scavengers of the metabolic and cardiovascular effects of endotoxin infusion in conscious rats. Circ. Shock, 19, 429-439.

22) Novotny, M. J., Laughlin, M. H., and Adams, H. R. (1988): Evidence for lack of importance of oxygen free radicals in Escherichia coli endotoxemia in dogs. Am. J. Physiol., 254, H954-H962.

23) Redl, H., Lieners C., Bahrami, S., Schlag, G., Van Bebber, I. P. T., and Goris, R. J. A. (1990): SOD in rat models of shock and organ failure, in Antioxidants in Therapy and Preventive Medicine, ed. I. Emerit, Plenum Press, New York, pp. $17-27$.

24) Kilbourn, R. G., and Griffith, O. W. (1992): Overproduction of nitric oxide in cytokine-mediated and septic shock. J. Natl. Can. Inst., 84, 827-831.

25) Moncada, S., Palmer, R. M. J., and Higgs, E. A. (1991): Nitric oxide physiology, pathophysiology, and pharmacology. Pharmacol. Rev., 43, 109-142.

26) Kilbourne, R. G., Jubran, A., Gross, S. S., Griffith, O. W., Levi, R., Adams, J., and Lodato, R. F. (1990): Reversal of endotoxin-mediated shock by $\mathrm{N}^{\mathrm{G}}$-methyl-Larginine, an inhibitor of nitric oxide synthesis. Biochem. Biophys. Res. Commun., 172, 1132-1138.

27) Nava, E., Palmer, R. M. J., and Moncada, S. (1991): Inhibition of nitric oxide synthesis in septic shock: how much is beneficial? Lancet, 338, 1555-1557.

28) Kilbourn, R. G., Gross, S. S., Jubran, A., Adams, J., Griffith, O. W., Levi, R., and Lodato, R. F. (1990): $\mathrm{N}^{\mathrm{G}}$-methyl- L-arginine inhibits tumor necrosis factor induced hypotension: implications for the involvement of nitric oxide. Proc. Natl. Acad. Sci., (USA) 87, 3629-3632.

29) Rees, D. D., Cellek, S., Palmer, R. M. J., and Moncada, S. (1990): Dexamethasone prevents the induction by endotoxin of a nitric oxide synthase and the associated effects on vascular tone: an insight into endotoxin shock. Biochem. Biophys. Res. Commun., 173, 541-547.

30) Petros, A., Bennett, D., and Vallance, P. (1991): Effect of nitric oxide synthase inhibitors on hypotension in patients with septic shock. Lancet, 338, 1557-1558.

31) Hotchkiss, R. C., Karl, I. E., Parker, J. L., and Adams, H. R. (1992): Inhibition of NO synthesis in septic shock. Lancet, 339, 434-435.

32) Silva, A. T., Bayston, K. F., and Cohen, J. (1990): Prophylactic and therapeutic effects of a monoclonal antibody to tumor necrosis factor-alpha in experimental gram negative shock. J. Infect. Dis., 162, 421-427. 
33) Acki, N., Johnson, G., and Lefer, A. M. (1990): Beneficial effects of two forms of NO administration in feline splanchnic occlusion shock. Am. J. Physiol., 2587, G275-G281.

34) Cohen, J., and Silva, A. (1992): NO inhibitors and septic shock. Lancet, 339, 751.

35) Anonymous (1991): Nitric oxide in the clinical arena. Lancet, 338, 1560-1562.

36) Weiland, J. E., Davis, W. B., Holter, J. F., Mohammed, J. R., Dorinsky, P. M., and Gadek, J. E. (1986): Lung neutrophils in the adult respiratory distress syndrome. Clinical and pathophysiologic significance. Am. Rev. Resp. Dis., 133, $218-225$

37) Suzuki, Y., Toshimori, T., Heimer, D., Wang, W., Ross W. G., Sussman, H. H., and Raffin, T. A. (1992): Polyethylene glycol-conjugated superoxide dismutase attenuates septic lung injury in guinea pigs. Am. Rev. Resp. Dis., 145, 388-393.

38) Pacht, E. R., Timmerman, A. P., Lykens, M. G., and Merola, A. J. (1991): Deficiency of alveolar fluid glutathione in patients with sepsis and the adult respiratory distress syndrome. Chest, 100, 1397-1403.

39) Tate, R. M., Vanbenthuysen, K. M., Shasby, D. M., McMurty, I. F., and Repine, J. E. (1982): Oxygen-radical-mediated permeability edema and vasconstriction in isolated perfused rabbit lungs. Am. Rev. Resp. Dis., 126, 802-806.

40) Johnson, K. J., Fantone, J. C., Kaplan, J., and Ward, P. A. (1981): In vivo damage of rat lungs by oxygen metabolites. J. Clin. Invest. 67, 983-993.

41) Baldwin, S. R., Simon, R. H., Grum, C. M., Ketai, L. H., Boxer, L. H., and Devall, L. J. (1986): Oxidant activity in expired breath of patients with adult respiratory disress syndrome. Lancet, 1, 11-14.

42) Sznajder, J. I., Fraiman, A., Hall, J. B., Sanders, W., Schmidt, G., Crawford, G., Nahum, A., Factor, P., and Wood, L. D. (1989): Increased hydrogen peroxide in the expired breath of patients with acute hypoxemic respiratory failure. Chest, $\mathbf{9 6}$, 606-612.

43) Dworkin, B. M., Rosenthal, W. S., Wormser, G. P., and Weiss, L. (1986): Selenium deficiency in the acquired immunodeficiency syndrome. JPEN, 10, 405-407.

44) Zazzo, J. F., Chals, J., Lafont, A., Camus, F., and Chappuis, P. (1987): Is nonobstructive cardiomyopathy in AIDS a selenium deficiency-related disease? JPEN, 12, 537-538.

45) Eck, J.- P, Gmunder, H., Hartmann, M., Petzoldt, D., Daniel, V., and Droge, W. (1989): Low concentations of acid-soluble thiol (cysteine) in the blood plasma of HIV-1 infected patients. Biol. Chem. Hoppe-Seyler, 370, 101-108.

46) Buhl, R., Holroyd, K. J., Mastrangeli, A., Cantin, A. M., Jaffe, H. A., Wells, F. B., Saltini, C., and Crystal, R.G. (1989): Systemic glutathione deficiency in symptom free HIV seropositive individuals. Lancet, 2, 1294-1298.

47) Roederer, M., Staal, F. J. T., Osada, H., Herzenberg, L. A., and Herzenberg, L. A. (1991): CD4 and CD8 T cells with high intraceullular glutathione levels are selectively lost as the HIV infection progresses. Int. Immunol., 3, 933-937.

48) Roederer, M., Ela, S. W., Staal, F. J. T., Herzenberg, L. A., and Herzenberg, L. A. (1992): N-acetylcysteine: A new approach to anti-HIV therapy. AIDS Res. 
Hum. Retroviruses, 8, 209-217.

49) Poli, G., Bressler, P., Kinter, A., Duh, E., Timmer, W. C., Rabson, A., Justement, J. S., Stanley, S., and Fauci, A. S. (1990): Interleukin 6 induces human immunodeficiency virus expression in infected monocytic cells alone and in synergy with tumor necrosis factor-alpha by transcriptional and post-transcriptional mechanisms. J. Exp. Med., 172, 151-158.

50) Staal, F. J. T., Ela, S. W., Roederer, M., Anderson, M. T., Herzenberg, L. A., and Herzenberr, L. A. (1992): Glutathione deficiency and human immunodeficiency virus infection. Lancet, 339, 909-912.

51) Nabel, G., and Baltimore, D. (1987): An inducible transcription factor activates expression of human immunodeficiency virus in $\mathrm{T}$ cells. Nature 326, 711-713.

52) Schreck, R., Rieber P., and Baeuerle, P. A. (1991): Reactive oxygen intermediates as apparently widely used messengers in the activation of the NF-kappaB transcription factor and HIV-1. EMBO J. 10, 2247-2258.

53) Staal, F. J. T., Roederer, M., L. A., and Herzenberg, L. A. (1990): Intracellular thiols regulate activation of nuclear factor $k \mathrm{~B}$ and transcription of human immunodeficiency virus. Proc. Natl. Acad. Sci. USA, 87, 9943-9947. 